\title{
Modern View on the Problem of Bronchial Asthma Comorbidity
}

\author{
Mykola Ostrovskyy ${ }^{1,}$, Lilia Todoriko $^{2}$, Oleksandr Varunkiv ${ }^{1}$, Iryna Makoida ${ }^{1}$, Halyna Korzh ${ }^{1}$, \\ Mariana Kulynych-Miskiv ${ }^{1}$, Liudmyla Babliuk ${ }^{1}$, Olha Molodovets ${ }^{1}$ \\ ${ }^{1}$ Department of Phthisiology and Pulmonology with the Course of Occupational Diseases, Ivano-Frankivsk National Medical University, \\ Ivano-Frankivsk, Ukraine \\ ${ }^{2}$ Department of Phthisiology \& Pulmonology, Higher State Educational Establishment “Bukovinian State Medical University”, Chernivtsi, \\ Ukraine
}

Email address:

mykola.m.ostrovskyy@gmail.com (M. Ostrovskyy)

${ }^{*}$ Corresponding author

\section{To cite this article:}

Mykola Ostrovskyy, Lilia Todoriko, Oleksandr Varunkiv, Iryna Makoida, Halyna Korzh, Mariana Kulynych-Miskiv, Liudmyla Babliuk, Olha Molodovets. Modern View on the Problem of Bronchial Asthma Comorbidity. American Journal of Internal Medicine. Special Issue: New Approaches to Manage Difficult-to-Control, Severe Asthma. Vol. 8, No. 2, 2020, pp. 51-56. doi: 10.11648/j.ajim.20200802.11

Received: December 24, 2019; Accepted: February 4, 2020; Published: March 2, 2020

\begin{abstract}
We have examined 40 patients with bronchial asthma (BA) based at the municipal non-profit enterprise "IvanoFrankivsk Regional Phthisiopulmonology Center of the Ivano-Frankivsk Regional Council" (Center for Pulmonary Diseases). The patients were randomized into two groups: Group I (17) involved patients with BA, Group II included (23) patients suffering from BA with obesity. The goal of the research work was to analyze the manifestations of clinical symptomatology, manageability of BA, state of pulmonary function, level of leptin in relation to mono- or polymorbidity. The research findings show the decrease in asthma manageability level in patients with combined pathology, particularly BA + obesity + gastroesophageal reflux disease (GERD), significant negative correlation $(r=-0.95, p<0.001)$ between FEV1 and ACQ-5 data in patients suffering from BA with obesity and GERD. The frequency of nocturnal asthma attacks, heartburn, and sleep disorders tended to increase in patients with comorbid conditions. Features of changes in the concentration of leptin depending on the detected pathology is characterized by the following data. The level of leptin in patients with BA with and without obesity is 4.82 times higher as compared to patients with combined pathology $(45.28 \pm 10.42 \mathrm{ng} / \mathrm{ml})$, and is significantly different $(\mathrm{p}<0.05)$ from the indices observed in patients with normal body weight $(9.40 \pm 1.52 \mathrm{ng} / \mathrm{ml})$.
\end{abstract}

Keywords: Bronchial Asthma, Obesity, Spirometry, Polymorbidity

\section{Introduction}

Throughout its existence, humankind is on its increase and constantly develops with the aim of positive entropy. Gradually improving everything around us, we have reached the globalization of society. In recent decades, a considerable breakthrough has been made in many fields of activity, including medicine. Modern technologies, up-to-date methods of diagnosis and treatment of many diseases which seemed to be insurmountable have had a significant impact on the expectancy and quality of life. Regrettably, the development of new diseases keeps pace with humanity. According to figures provided by the experts, the incidence rate of these ailments is rapidly increasing, leading to global economic burden, which amounts to trillions of dollars. Although, the medical community has concentrated its efforts on fighting infectious diseases such as HIV/AIDS, malaria and tuberculosis, non-communicable diseases have "relatively obscurely come to the fore in developing countries and currently become a global problem" [5, 8].

In 2011, the United Nations announced the worldwide epidemic of chronic non-communicable diseases (CNCDs). By 2030, WHO forecasts an increase of mortality rates from non-communicable diseases up to 52 million people per year $[6,7]$. The problem is aggravated by the combination of some CNCDs, the so-called comorbid conditions. The concurrent onset of two or more causal, competitive or 
intercurrent diseases leads to poor quality of life, increases costs for diagnostic examination and treatment, extends the period and frequency of hospital admissions. Thuswise, nosological syntropy, as a type of polymorbidity, when some diseases simultaneously «follow each other, seek to combine or set the ground for each other», is the cornerstone modern physicians have to fight with $[5,10]$.

What does the patient want: treatment of symptoms (occasionally the relief of acute medical conditions) or control over the disease? A person, conscious of his/her illness, seeks to live a full-on life, and medicine must indicate the way to achieve it.

Bronchial asthma (BA) is one of the dangerous noncommunicale epidemics, currently overwhelming the humankind. According to various sources, its worldwide prevalence rate ranges from $1 \%$ to $18 \%$ and is steadily increasing $[1,4,7]$. The latest GINA (Global Initiative for Asthma) recommendations state that the treatment endpoints for patients with BA are to achieve and maintain control over the clinical manifestations of the disease for a long time in terms of low-dose ICS/formoterol regimen as a basic therapy or on demand $[4,9,11]$. The epidemiological studies show that, averagely, half of the patients are unable to achieve or maintain long-term BA control. Often, asthma is not diagnosed, especially in case of non-classical clinical manifestations or severe course, disguising itself as COPD, chronic bronchitis, pathology of the upper respiratory tract, gastroesophageal reflux disease $[2,7,9,13,14]$. The reason is the heterogeneity of bronchial asthma by its mechanisms, manifestations, clinical course, and also a significant increase of its incidence rates if comorbid with obesity. Over $54.6 \%$ of Ukrainians are overweight, and $24.6 \%$ are diagnosed with obesity. Significant prevalence of these risk factors is associated with unhealthy diet where carbohydrates prevail and low physical activity $[1,6,10,15]$.

Modern view on the function of adipose tissue, as a depot of energy yielding material, endocrine and paracrine organ, as well as the producer of adipokines (leptin, regulation of inflammatory processes, immune homeostasis), gives understanding of the negative synergy between bronchial asthma, its management and obesity. Leptin is considered to be responsible for adequate ventilatory stimulus in response to increased respiratory function in obesity, while its deficiency may result in alveolar hypoventilation $[5,6,13]$. Considering the fact that in many cases of alveolar hypoventilation, leptin levels are high, made it possible to recognize another branch of pathogenesis - resistance to leptin. Moreover, leptin affects the T-cell immune response and stimulates the proliferation of $\mathrm{T}$-helper cells that promote the synthesis of proinflammatory cytokines [6, 10, 14]. The clinical picture of asthma in overweight patients, is characterized not only by more severe course and poor control, but also by an inadequate response to regulated base line therapy $[4,7,9]$. Low efficacy of base line therapy and tolerance to bronchodilators requiring increased doses of drugs, twice as frequent use of systemic glucocorticoids, are the evidence of decreased effectiveness of asthma control.
GINA guidelines insist on the importance of reducing excess weight in patients with asthma in order to relieve symptoms, improve ventilatory capacity of the lungs and reduce the manifestations of inflammation [4, 7, 8, 11]. In addition, many studies have revealed the direct correlation between increased body weight and the presence of gastroesophageal reflux disease (GERD). "Pulmonary mask" is characterized by the development of bronchial obstruction on the background of GERD, chronic cough (especially at night), occasional sleep apnea, recurrent pneumonia, pulmonary fibrosis. Osler is known to be the first to associate asthma attack with pulmonary aspiration of gastric contents in 1892 . Nowadays, GERD is considered as a trigger for bronchial asthma (BA). The presence of GERD may also explain the nocturnal attacks of asthma, when patients are in a horizontal position, which contributes to the regurgitation of gastric contents into the esophagus. Numerous studies have shown the association between BA and GERD. The investigations show that heartburn occurs in patients with asthma in $70 \%$ of cases, thus the daytime episodes occur in $20 \%$ of cases, while day and night-time episodes occur in $50 \%$ of cases. The presence of GERD is observed in $33-90 \%$ of patients with asthma, however, pathological gastroesophageal refluxes have no "esophageal" manifestations in $25-30 \%$ of cases [2, $3,12]$.

\section{Materials and Methods}

We have examined 40 patients with bronchial asthma (BA) based at the municipal non-profit enterprise "IvanoFrankivsk Regional Phthisiopulmonology Center of the Ivano-Frankivsk Regional Council" (Center for Pulmonary Diseases). The inclusion criteria involved patients whose FEV1 prior to the use of bronchodilators was $40-80 \%$. Additionally, no bacterial or viral infections, and no systemic glucocorticosteroids were reported for one month prior to inclusion. Patients' complaints, history of allergies, past life and medical history were carefully recorded. All the patients have undergone diagnostic and general clinical examinations. The ACQ-5 test, which consisted of 5 questions with the 6point response scale, was used to determine the level of BA manageability. The overall ACQ-5 score was calculated as the arithmetic mean of 5 answers. Group1-a: $<0.5-0.75-$ well-controlled BA. Group 2-a: 0.75-1.5 - partly controlled BA. Group 3: $>1.5$ - uncontrolled asthma. The respiratory function (RF) was investigated using a spirograph "Spirocom"; the parameters were compared by the patients' age, sex and body weight indices. Body mass index (BMI) was identified as Quetelet index. All the patients were divided into two groups in order to determine the features of the BA course accompanied by obesity. Group I involved 17 BA patients with normal body weight $\left(\mathrm{BMI} \leq 25 \mathrm{~kg} / \mathrm{m}^{2}\right)$; group II included 23 patients suffering from BA with obesity $\left(B M I \geq 30 \mathrm{~kg} / \mathrm{m}^{2}\right)$, where IIa group involved 14 patients with BA+obesity, IIb group involved 9 patients with BA+obesity+GERD. The level of leptin was measured using the «DRG Leptin ELISA» kit (Germany). The method is 
based on the "sandwich principle". The microtiter wells are covered with monoclonal antibodies specific to the unique antigenic site on the leptin molecule. The aliquot of the sample of patient with endogenous leptin is incubated in a well with a specific biotinylated monoclonal anti-leptin antibody and a "sandwich complex" is formed. After incubation, the unbound material is washed and the conjugate (streptavidin-peroxidase enzyme complex) is added to detect bound leptin. After the substrate is added, the intensity of the resulting staining is determined, which is proportional to the concentration of leptin in the sample.

Variable-based statistical method was used to objectively analyze the confidence of investigation findings on the Pentium II personal computer using "Statistica 8.0" software package and "Microsoft Excel" statistical function package. The parametric data were reported as $\mathrm{M} \pm \mathrm{m}$. In-group dynamics was estimated using the paired Student t-test. Statistical analysis involved the calculation of arithmetic mean $(\mathrm{M})$, standard deviation $(\delta)$, standard error of the arithmetic mean value $(m)$, and probability of the difference of the investigation findings (p). Pearson's correlation coefficient (r) was defined in order to determine the correlation between specific indicators.

\section{Results}

Female patients prevailed in groups I and II, 64.7\% and $69.6 \%$ respectively. The average age of patients, duration and frequency of asthma exacerbations made up: Group I - (39.17 $\pm 1.23)$ years, $(11.15 \pm 1.24)$ years, $(1.70 \pm 0.34)$ times/year; Group II - (48.24 \pm 1.26$)$ years, $(13.34 \pm 1.32)$ years, $(3.46 \pm$ $0.35)$ times/year. The body mass index (BMI) of patients from group I $(21.34 \pm 0.34)$ corresponded to the parameters of normal body weight, while in group II patients $(34.28 \pm 0.42)$ it pointed to obesity. Hereditary allergic anamnesis of patients with isolated asthma was aggravated in $17.6 \%$ of cases, while in comorbidity it made up $39.1 \%$. The results of the survey and patients' charts review showed that bronchial asthma was detected on the background of already existing obesity in 11 (47.8\%) patients from group II, while 9 (39.1\%) patients were diagnosed with GERD along with comorbidity.

Table 1. Complaints of patients with asthma depending on comorbid obesity.

\begin{tabular}{|c|c|c|c|c|}
\hline \multirow{3}{*}{ Complaints } & \multicolumn{4}{|l|}{ Groups of patients } \\
\hline & \multicolumn{2}{|l|}{ BA $(n=17)$} & \multicolumn{2}{|l|}{ BA+Obesity $(n=23)$} \\
\hline & Absolute number & $\%$ & Absolute number & $\%$ \\
\hline 1 & 2 & 3 & 4 & 5 \\
\hline Cough & 17 & 100 & 23 & 100 \\
\hline Expectoration & 11 & 64.7 & 18 & 78.3 \\
\hline Exertional dyspnea & 17 & 100 & 23 & 100 \\
\hline Dyspnea at rest & 8 & 47.1 & 19 & 82.6 \\
\hline Heartburn & 5 & 29.4 & 19 & 82.6 \\
\hline Sleep disorders & 12 & 70.5 & 23 & 100 \\
\hline
\end{tabular}

The distribution of complaints frequency (Table 1) shows that cough and exertional dyspnea were observed in $100 \%$ of the examined patients, though, dyspnea at rest, was observed twice as often in patients with obesity $(82.6 \%)$ than without it (47.1\%). The frequency of nocturnal asthma attacks, heartburn, and sleep disorders tended to increase in patients with comorbid conditions.

The level of leptin in patients with BA with and without obesity is 4.82 times higher as compared to patients with combined pathology $(45.28 \pm 10.42 \mathrm{ng} / \mathrm{ml})$, and is significantly different $(\mathrm{p}<0.05)$ from the indices observed in patients with normal body weight $(9.40 \pm 1.52 \mathrm{ng} / \mathrm{ml})$.

Table 2. The indices of RF and ACQ-5 in patients with BA suffering from obesity and those with normal body weight.

\begin{tabular}{|c|c|c|c|c|}
\hline \multirow{3}{*}{ RF index,\% } & \multirow{2}{*}{ Group I $(n=17)$} & \multicolumn{2}{|c|}{ Group II $(n=23)$} & \multirow{3}{*}{$\mathbf{p}$} \\
\hline & & IIa $(n=14)$ & IIb $(n=9)$ & \\
\hline & 1 & 2 & 3 & \\
\hline FVC & $92.18 \pm 5.61$ & $83.39 \pm 7.92$ & & $\begin{array}{l}\text { p1-2>0.05 } \\
\text { p1-2<0.05; }\end{array}$ \\
\hline FEV1 & $55.58 \pm 2.42$ & $46.86 \pm 2.62$ & $40.78 \pm 1.19$ & $\begin{array}{l}\text { p1-3<0.001; } \\
\text { p2-3<0.05 } \\
\text { p1 }-2>0.05\end{array}$ \\
\hline $\mathrm{FEV}_{1} / \mathrm{FVC}$ & $67.58 \pm 4.27$ & $61.07 \pm 1.46$ & $56.11 \pm 1.28$ & $\begin{array}{l}\text { p1-3<0.05; } \\
\text { p2-3<0.05 }\end{array}$ \\
\hline $\mathrm{ACQ}$ & $2.1 \pm 0.28$ & $3.5 \pm 0.51$ & $3.5 \pm 0.51$ & $\mathrm{p} 1-2<0.05$ \\
\hline
\end{tabular}

Table 2 shows that FVC indices in patients from groups I and II did not differ significantly $(\mathrm{p}>0.05)$; the FEV1 indices in patients from group I were $15.7 \%$ higher as compared to the same indices in patients from subgroup IIa $(\mathrm{p}<0.05)$ and $26.6 \%$ higher as compared to patients from subgroup $\mathrm{IIb}$ $(\mathrm{p}<0.001)$. The $\mathrm{FEV}_{1} / \mathrm{FVC}$ index in patients from group I was $9.6 \%$ higher as compared to the same index in patients from subgroup IIa $(p>0.05)$ and $17.0 \%$ higher than in patients from IIb subgroup $(\mathrm{p}<0.05)$. The ACQ-5 index in patients from group II was 1.7 times higher than the same index in patients from group I $(\mathrm{p}<0.05)$. 


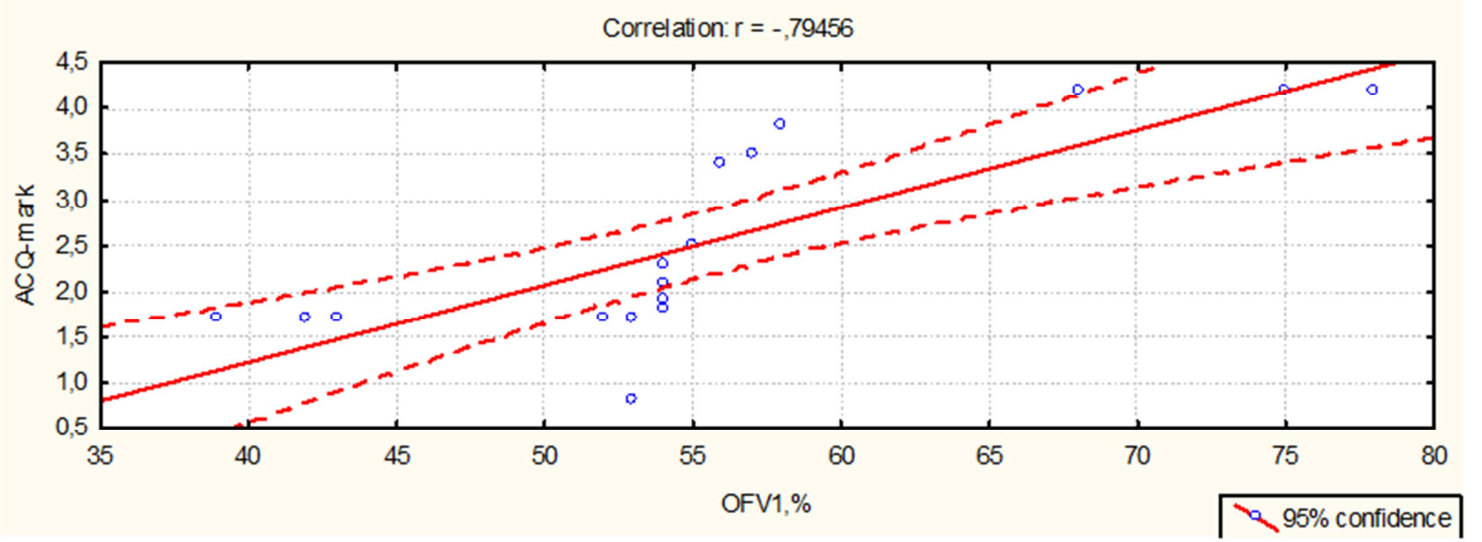

Figure 1. Correlation between FEVIindices and ACQ-5 data in patients with bronchial asthma without obesity

Figure 1 shows mean negative correlation between the indices of FEV1 and ACQ-5 data in patients suffering from BA without obesity $(\mathrm{r}=-0.79, \mathrm{p}<0.05)$.

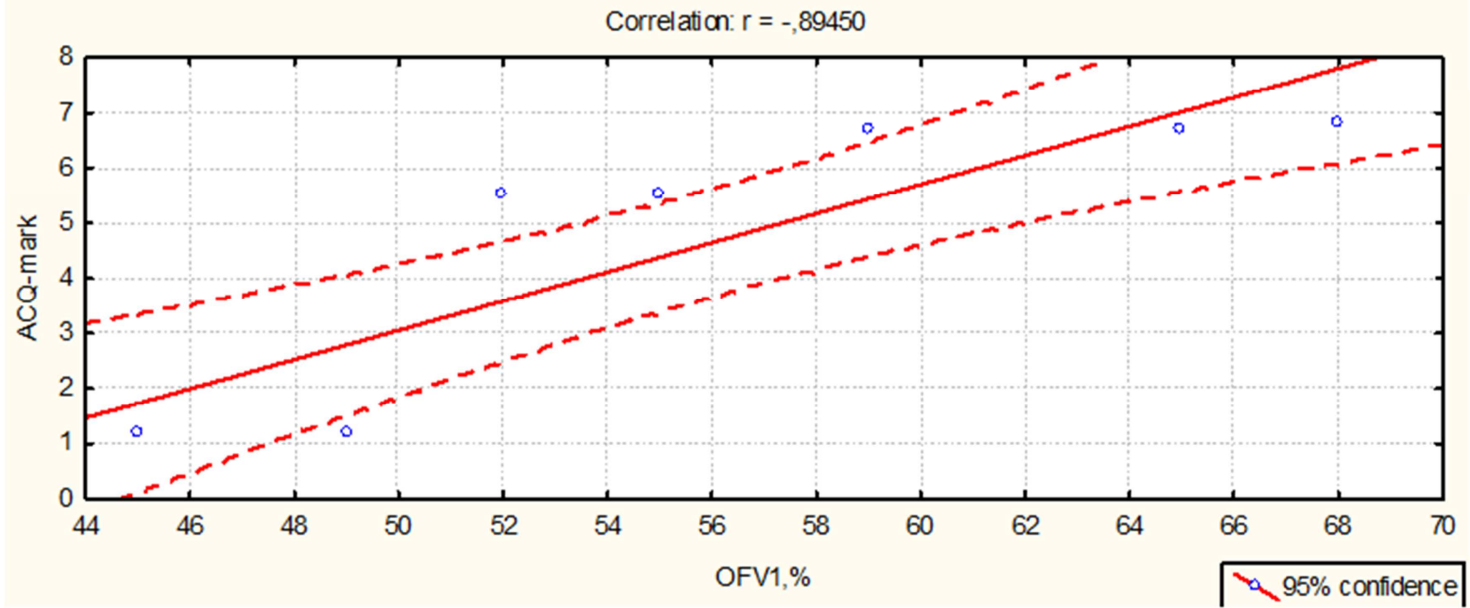

Figure 2. Correlation between FEVIindices and ACQ-5 data in patients suffering from bronchial asthma with obesity without GERD

Figure 2 shows strong negative correlation between the indices of FEV1 and ACQ-5 data in patients suffering from BA with obesity without GERD $(\mathrm{r}=-0.89, \mathrm{p}<0.01)$

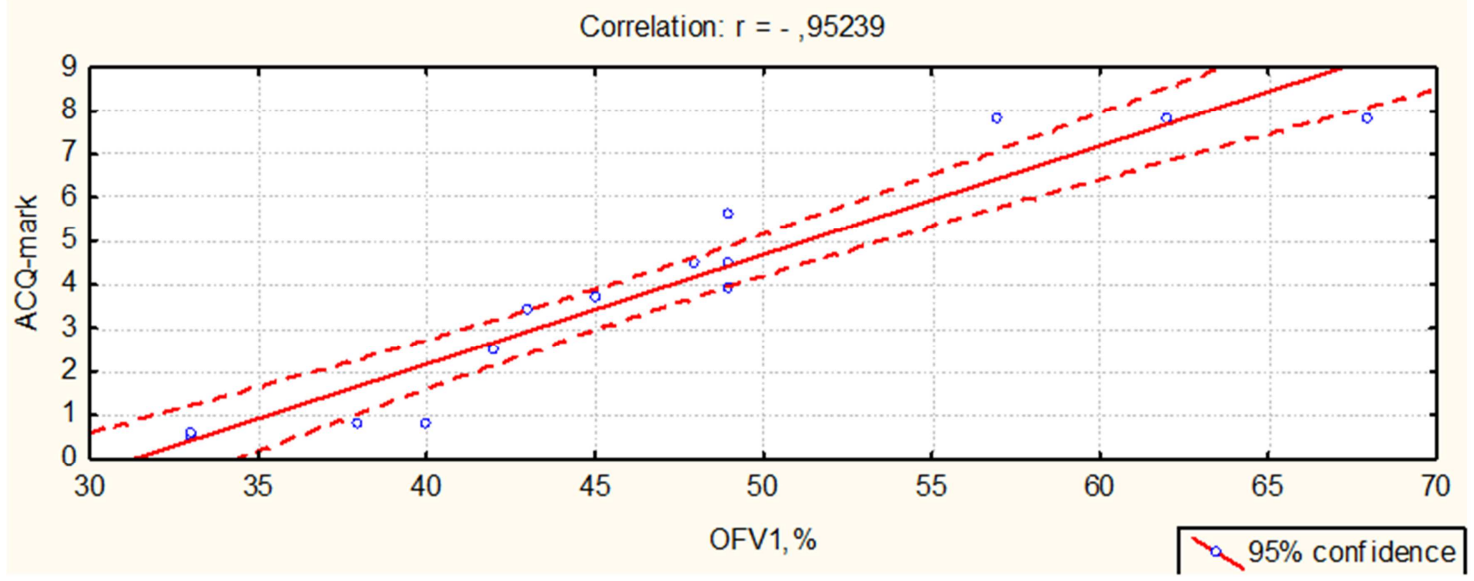

Figure 3. Correlation between FEVIindices and ACQ-5 data in patients suffering from bronchial asthma with obesity and GERD.

Figure 3 shows strong negative correlation between the indices of FEV1 and ACQ-5 data in patients suffering from
BA with obesity and GERD ( $\mathrm{r}=-0.95, \mathrm{p}<0.001)$. 


\section{Discussion}

Bronchial asthma still remains not only an incurable, but also poorly controllable disease. A large number of investigations are aimed at studying the combination of asthma with diseases that complicate its clinical course and manageability. Scientists state that BA combined with obesity is one of the most common types of comorbidity [ 1 , $2,5,10]$. Patients with the above-mentioned comorbidity have significantly low rates of asthma control. Asthma control involves minor symptoms, absence of night-time attacks, minimal use of short-acting $\beta 2$ agonists or no need for their use, high tolerability of strenuous activities. If one or two of these criteria are absent, asthma is considered to be partly controled $[4,7,9]$. Our survey of patients shows similar data: the intensity and frequency of complaints in patients with comorbidity is more pronounced as compared to monopathology. Dyspnea at rest was observed almost twice as often in patients with obesity $(82.6 \%)$, than in patients without it $(47.1 \%)$. The incidence of nocturnal episodes of asthma and sleep disorders tended to increase in patients with comorbid conditions. Hereditary allergic anamnesis was twice more often aggravated in patients with comorbidity. The ACQ-5 test data shows that asthma was not controlled in both groups of patients. The ACQ index in patients from group II was 1.7 times higher than the same indicator in patients from group I $(\mathrm{p}<0.05)$.

The study findings show that nocturnal attacks of asthma occurred 1.6 times more often in patients from group II than in group I ( $91.3 \%$ vs $58.8 \%)$ patients, while the symptoms of heartburn were 2.8 times more common. 9 patients (39.1\%) from group II were diagnosed with GERD along with the comorbidity. The obtained study results confirm the opinion of a number of scientists [8] about the correlation between BA and GERD. The study revealed that heartburn occurs in $70 \%$ of cases among the patients with asthma, including daytime symptoms - in $20 \%$, and day and night-time symptoms - in $50 \%$ of cases. The presence of GERD was observed in $33-90 \%$ of patients with asthma, however, pathological gastroesophageal refluxes have no "esophageal" manifestations in $25-30 \%$ of cases.

The study of respiratory function shows the decrease of FEV1 indices in all the patients, while its indices are four times more prominent in patients with polymorbidity (IIb subgroup) $(26.6 \%)(\mathrm{p}<0.001)$ as compared to the indices recorded in group I patients. $\mathrm{FEV}_{1} / \mathrm{FVC}$ indices in patients from group I were $9.6 \%$ higher than the same indices recorded in patients from IIa subgroup ( $p>0.05)$ and $17.0 \%$ higher as compared to the patients from subgroup $\mathrm{IIb}$ $(p<0.05)$. The FVC indices recorded in patients from groups I and II were not significantly different $(p>0.05)$. Our findings have single-vector orientation with the research data of a number of other scientists $[2,5,12]$. GERD, the incidence of which increases with the increase of body mass index, is associated with high bronchial hyperactivity. Hydrochloric acid reflux into the esophagus leads to vagus nerve stimulation and bronchospasm. Micro-aspiration of acidic content into the bronchi also supports the inflammatory process leading to bronchoconstriction.

In the course of investigation we have discovered correlation relationships of varying intensity between FEV1 decrease and asthma control results. Thus, the study revealed mean negative correlation $(r=-0.79, p<0.05)$ in patients from group I, and strong negative correlations in patients from subgroups IIa and IIb $(\mathrm{r}=-0.89, \mathrm{p}<0.01)$ and $(\mathrm{r}=-0.95$, $\mathrm{p}<0.001$ ), respectively.

\section{Conclusion}

The obtained findings point to the relevance of our approach to study, treatment and control of especially severe asthma, as an etiologically multifactorial disease with frequent polymorphism. The latter, in its turn, may be both the predictor and cooperator in the development of severe, hardly curable and uncontrolled pathology. The combination of bronchial asthma with obesity is quite common example. Obesity keeps pace with the development of asthma leading to bronchial hyperactivity and decrease in respiratory excursion. Leptin production by adipose tissue is considered as one of the pathogenetic mechanisms contributing to the development of complications and aggravating the clinical course of asthma. Obesity also influences the respiratory function $(\mathrm{RF})$ : it reduces the forced expiratory volume in 1 second (FEV1) and forced vital capacity (FVC). Besides, the presence of GERD in people with overweight initiates increased bronchoconstriction, which may be a predictor of asthma or cause heartburn and asthma attacks especially at night. The study revealed the decrease in asthma control indices, especially in patients with polymorphism, and the presence of strong negative correlation $(\mathrm{r}=-0.95, \mathrm{p}<0.001)$ between control indices and FEV1. The above-mentioned results motivate the clinician to approach the treatment of BA as a pathology requiring complex therapy. The disease should not be treated symptomatically. The principles of disease manageability require more comprehensive means which may influence the state of bronchi, suppress inflammation, decrease body weight, improve the secretory and motile function of the stomach.

\section{Compliance with Ethics Requirements}

"The study was performed according to the requirements of the Statute of Ukrainian Association for Bioethics and the GCP norms (1992), requirements and norms of ICH GLP (2002), ethical standards in the Helsinki Declaration of 1975, as revised in 2008, typical ethics provisions of the Ministry of Public Health of Ukraine 66 dated February 13, 2006. The work was approved by Committee on Bioethics Questions of Regional Centre of Phthisiology and Pulmonology, IvanoFrankivsk, Ukraine (Protocol No 01.01/18 on January 11, 2018; chairmen - MD, Konyuska M. Y.)".

The authors declare no conflict of interest regarding this article:

"Informed consent was obtained from all the patients 
included in the study".

No funding for this study.

\section{References}

[1] Besh OM, Slaba OP, Radchenko OM. Controllability of bronchial asthma in patients with different body weight. Bulletin of problems of biology and medicine 2016 (Ukr); 1 (133): 123-126.

[2] Velichko VI, Wenger II, Platonov OM. Bronchial asthma, obesity and gastroesophageal reflux disease: possible mechanisms of correlation. Odesa Medical Journal 2013 (Ukr); 3 (137): 57-62.

[3] Dragomiretska NV, Zabolotna IB, Guscha SG, Kalinichenko MV, Yizha GM. New possibilities of treatment of refractory gastroesophageal reflex disease. Bulletin of problems of biology and medicine 2019 (Ukr); 2 (149): 138-141.

[4] Ostrovskyy MM. Changes to GINA 2019: new perspectives for Patient and Doctor. Asthma and Allergy 2019 (Ukr); 4: 6859.

[5] Suhan VS. Comorbid course of bronchial asthma and obesity. Ukraine. Nation's health 2018 (Ukr); 2 (49): 70-72.

[6] Fediv OI, Miller OB. Hypolipidemic therapy in the complex treatment of obesity-associated bronchial asthma, taking into account gene polymorphism. Young scientist 2017 (Ukr); 9 (49): 70-74.
[7] Feshchenko YI. Bronchial asthma, chronic obstructive pulmonary disease: a promising global strategy for management, advanced diagnostic methods, current approaches to therapy. Asthma and Allergy 2015 (Ukr); 4: 3842 .

[8] Feshchenko YI, Ostrovskyy MM, Varunkiv OI. Treatment of bronchial obstructive syndrome: beta-agonists. Ukrainian Pulmonary Journal 2019 (Ukr); 3 (105): 32-36.

[9] Feshchenko YI. New approaches to improving treatment of bronchial asthma in world practice. Asthma and Allergy 2019 (Ukr); 4: 56-57.

[10] Yashina LO. Bronchial asthma is an urgent problem today. Ukrainian Pulmonary Journal 2018 (Ukr); 3: 19-23.

[11] GINA 2018. Updated 2018. Available at: www.ginasthma.org.

[12] Boeckxstaens G. El-Serag H. B., Smout A. Symptomatic reflux disease: the present, the past and the future. BMJ 2014; 63 (7): 1185-1193.

[13] Boulet L. P. Asthma and obesity. Clin. Exp. Allergy 2013; 43 (1): $8-21$.

[14] Juel C. T., Ulrik C. S. Obesity and asthma: impact on severity, asthma control, and response to therapy. Respir Care 2013; 58 (5): $867-873$

[15] Zhao X M. R. FTO and obesity: mechanisms of association. Curr Diab Rep. 2014; 14 (5): 486. 